Supporting Information

\title{
Optical Excitation of Carbon Nanotubes Drives Localized Diazonium Reactions
}

\author{
Lyndsey R. Powell, Yanmei Piao, ${ }^{\dagger}$ YuHuang Wang*
}

Department of Chemistry and Biochemistry, University of Maryland, College Park, Maryland 20742, United States

*Correspondence should be addressed to Y.H.W. at yhw@umd.edu.

$\dagger$ Present address: Physical Measurement Laboratory, National Institute of Standards and Technology, Gaithersburg, Maryland 20899, United States

\section{METHODS}

Suspensions of chirality-enriched SWCNTs. Chirality-enriched SWCNT samples were separated from high-pressure carbon monoxide (HiPco) SWCNTs (Rice University, Lot 194.3) using an adapted gel chromatography procedure. ${ }^{1}$ We isolated $(6,5)-$ SWCNTs on a Sephacryl S-200 HR column (GE Healthcare). The SWCNTs used in these experiments were individually suspended by $1 \% \mathrm{~m} / \mathrm{v}$ SDS in $99.8 \% \mathrm{D}_{2} \mathrm{O}$ and the solution was adjusted to an optical density at $\mathrm{E}_{11}$ of $0.1 \mathrm{~A} / \mathrm{cm}$, corresponding to a carbon concentration of approximately $0.66 \mathrm{mg} / \mathrm{L} .^{1}$ This concentration is dilute such that inner-filter effects are negligible.

Optically-driven SWCNT functionalization. SWCNTs were covalently functionalized using $p$ nitrobenzenediazonium tetrafluoroborate, which was synthesized from its complementary aniline and nitrous acid as previously described. ${ }^{2}$ The successful synthesis of a high-purity diazonium salt was confirmed with Fourier transform infrared spectroscopy and ${ }^{1} \mathrm{H}$ nuclear magnetic resonance spectroscopy. The salts were stored at $0{ }^{\circ} \mathrm{C}$ until immediately before use. An aliquot of freshly prepared 
diazonium solution (of minimal volume to avoid disturbing the SWCNT concentration, typically $<0.3 \%$ increase in volume) was added to $(6,5)$-enriched solutions at the desired molar reactant ratio with stirring and briefly protecting it from light $(20 \mathrm{~min})$ to allow the aryldiazonium to diffuse to the SWCNT surface before the intended irradiation. Solutions were continuously irradiated with the monochromator-selected (10 $\mathrm{nm}$ bandpass) output from a $450 \mathrm{~W}$ Xenon arc lamp with stirring. The reactions were protected from ambient light.

Power density experiments. Absorptive neutral density filters (Edmund Optics) were placed in the beam path of the incident irradiation (monochromator-selected output from a $450 \mathrm{~W}$ Xenon arc lamp). Power density was measured with an optical power meter (Newport 1916-C) and silicon detector (Newport 918-SL-OD3).

Temperature dependence experiments. A circulating water bath stage (FL-1027, Horiba Jobin Yvon) was used to control the temperature of the reactant solution throughout irradiation. The solution temperature was measured with an immersion Surface Temperature Sensor and LabQuest 2 (Vernier).

Spectroscopic characterization of reaction. Fluorescence spectra and excitation-emission maps were collected on a HORIBA Jobin Yvon Nanolog Spectrofluorometer equipped with a $450 \mathrm{~W}$ Xenon arc lamp and a calibrated multichannel InGaAs array detector. Samples were monitored spectroscopically, infrequently to avoid light contamination, until defect PL peak intensity stabilized (approximately 3 and $250 \mathrm{~h}$ in the case of samples irradiated and protected from light, respectively). We note that the reported PL intensities are peak intensities. Once stable, ultraviolet/visible/near-infrared (UV-vis-NIR) absorption spectra were measured using a PerkinElmer Lambda 1050 UV-vis-NIR spectrophotometer equipped with a photomultiplier tube and a broadband InGaAs detector. Samples were prepared for Raman spectroscopy by precipitating SWCNTs in ethanol. Raman spectra were collected using a Horiba 
Jobin Yvon LabRAM Raman microscope (model ARAMIS) with the $632.8 \mathrm{~nm}$ output $\left(1.4 \mathrm{~mW} \mu \mathrm{m}^{-2}\right.$ ) of a helium-neon ion laser as the excitation source. 


\section{REFERENCES}

(1) Liu, H.; Nishide, D.; Tanaka, T.; Kataura, H. Large-Scale Single-Chirality Separation of SingleWall Carbon Nanotubes by Simple Gel Chromatography. Nat. Commun. 2011, 2.

(2) Piao, Y.; Meany, B.; Powell, L. R.; Valley, N.; Kwon, H.; Schatz, G. C.; Wang, Y., Brightening

of Carbon Nanotube Photoluminescence Through the Incorporation of $\mathrm{sp}^{3}$ Defects. Nat. Chem. 2013, 5, 840-845. 


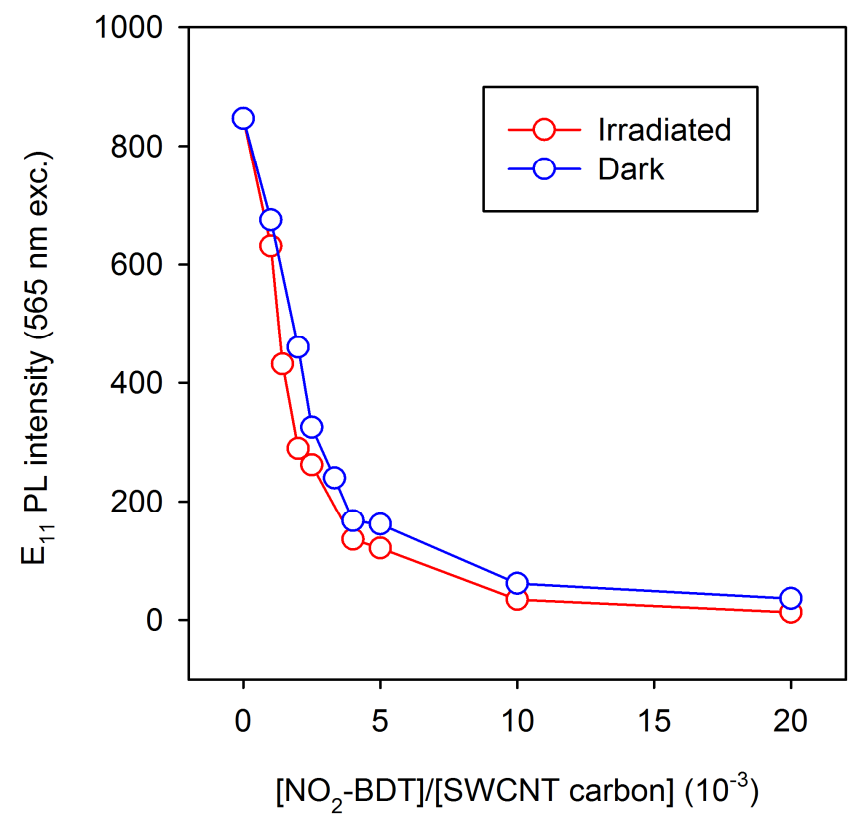

Figure S1. $\mathrm{E}_{11}$ PL intensity is quenched with increasing relative amounts of $\mathrm{NO}_{2}$-BDT added whether irradiated $(565 \mathrm{~nm})$ or protected from light. 

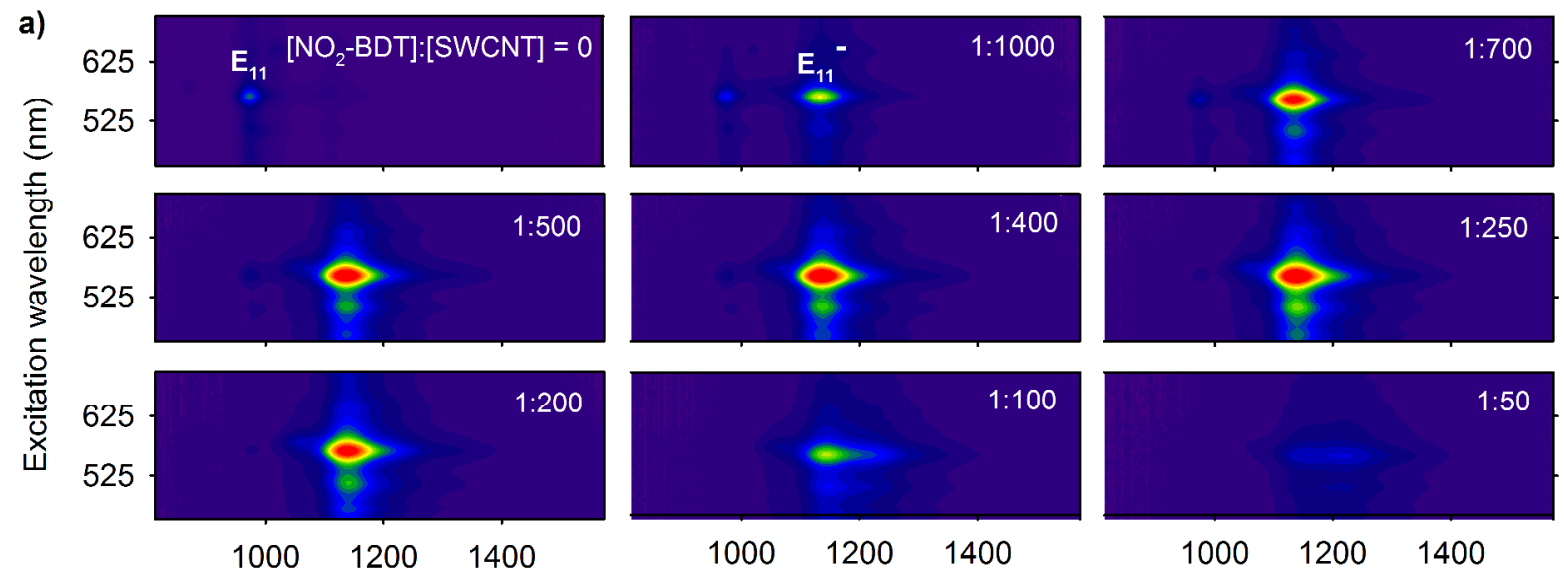
625 525 छ
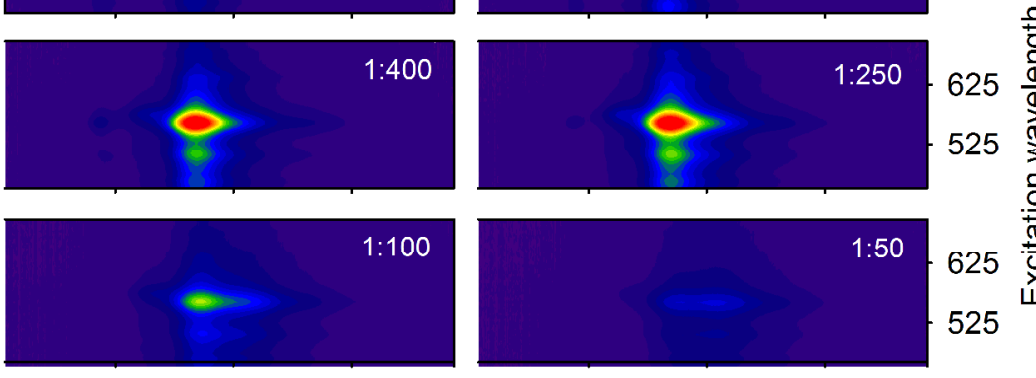

Emission wavelength $(\mathrm{nm})$
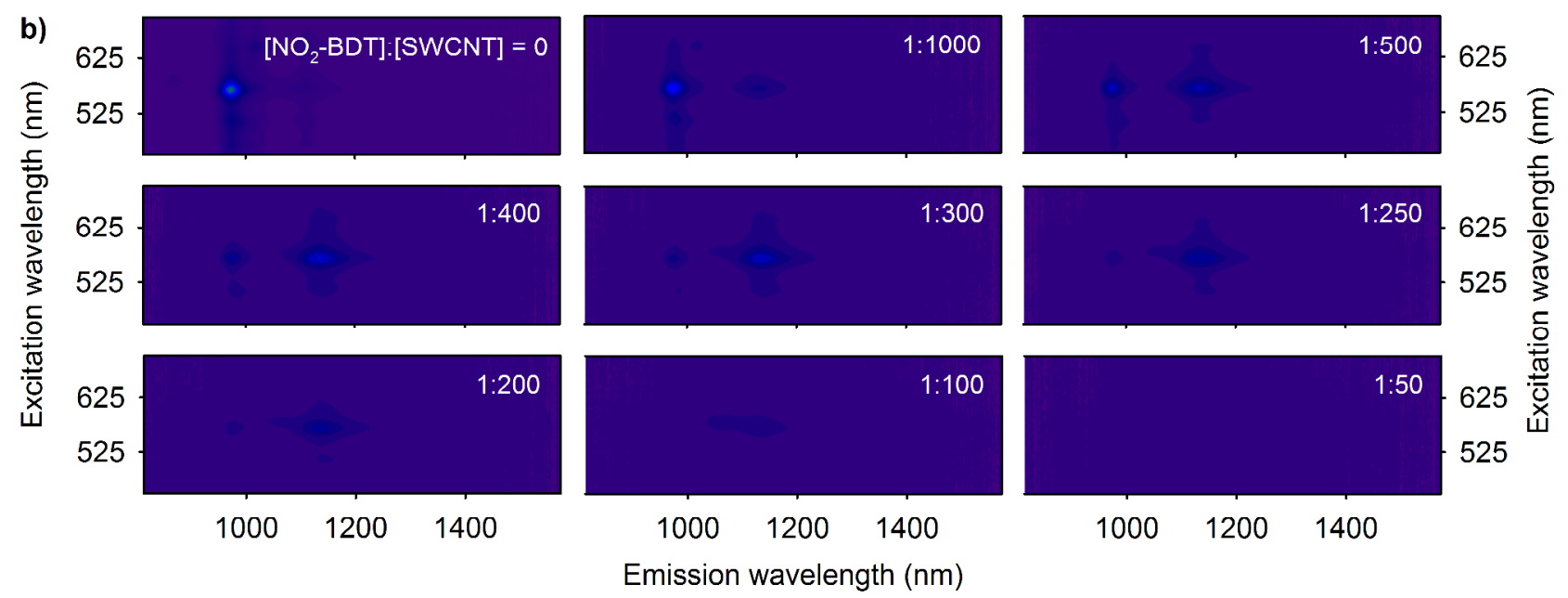

Figure S2. PL excitation-emission maps of $(6,5)$-enriched SWCNT solutions with various relative amounts of $\mathrm{NO}_{2}$-BDT. Functionalization was affected either under (a) $\mathrm{E}_{22}$-resonant irradiation (565 nm) or (b) protection from light. 

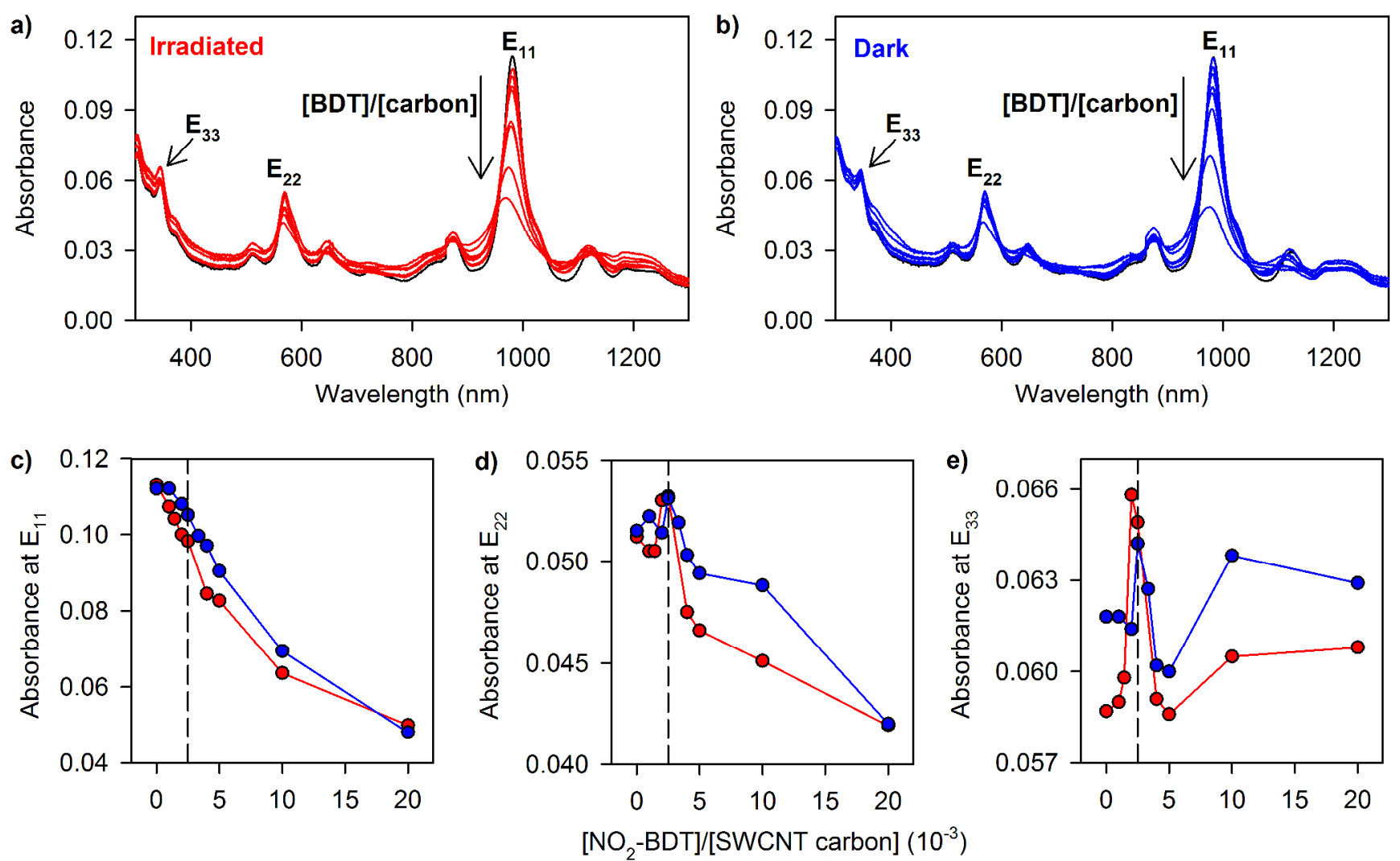

Figure S3. UV-vis-NIR optical absorption spectra (path length $=1 \mathrm{~cm})$ of $(6,5)$-enriched SWCNT solutions functionalized with various amounts of $\mathrm{NO}_{2}$-BDT either under (a) $\mathrm{E}_{22}$-resonant $(565 \mathrm{~nm})$ irradiation or (b) protection from light. Spectra were taken after defect $\mathrm{PL}$ at $\mathrm{E}_{11}{ }^{-}$was stable. Absorbance at (c) $E_{11}$, (d) $E_{22}$, and (e) $E_{33}$ are plotted versus relative ratios of reactants. The molar ratio which produced the most intense defect PL at $\mathrm{E}_{11}{ }^{-}$(see Figure 4) is indicated by the vertical dashed line in Figs $\mathrm{c}, \mathrm{d}$, and e for reference. 


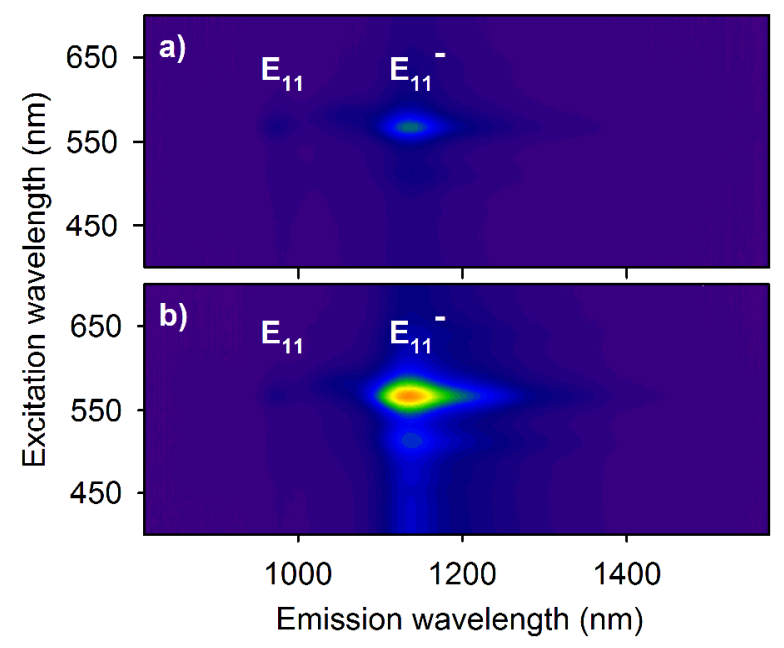

Figure S4. $\mathrm{E}_{22}$-resonant light $(565 \mathrm{~nm})$ is used to drive functionalization of a 6-week aged (top) sample of $(6,5)$ SWCNTs and $\mathrm{NO}_{2}$-BDT to generate bright defect PL at $\mathrm{E}_{11}{ }^{-}$(bottom) whose position is consistent with that of nitrobenzene moieties. 


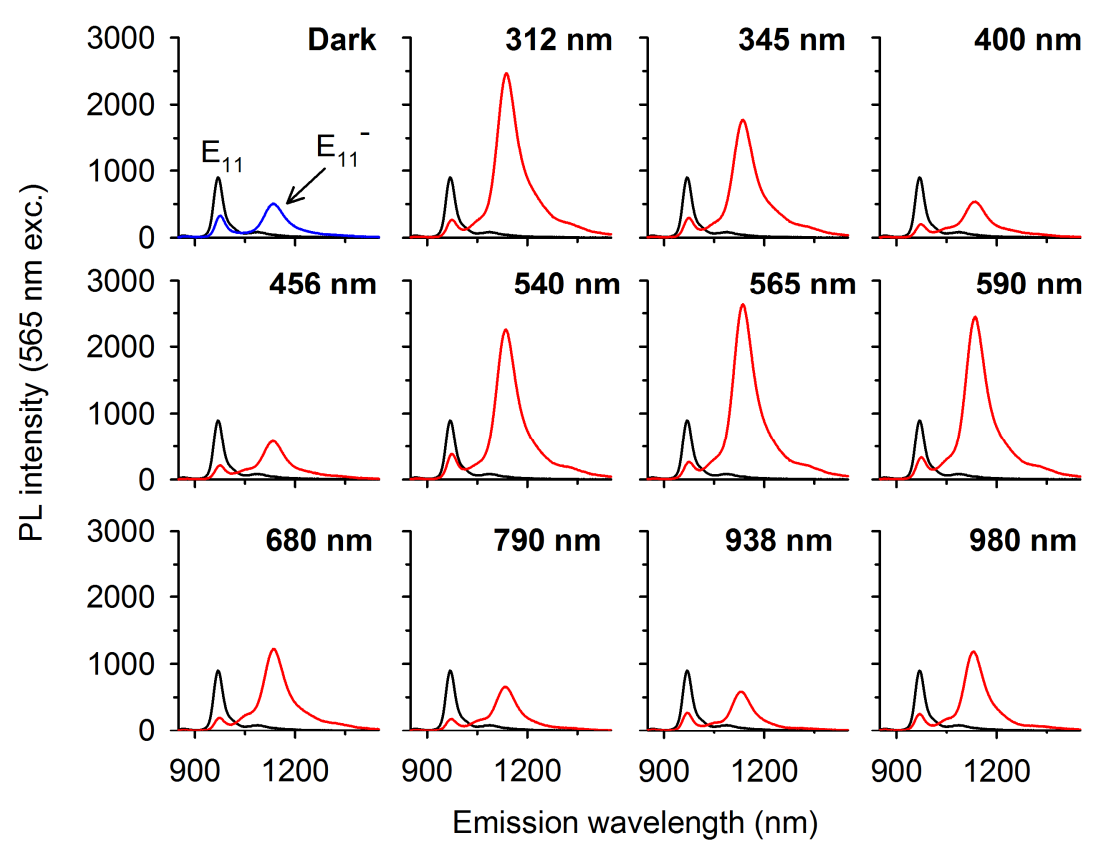

Figure S5. PL emission spectra (blue, red) of all samples used in wavelength dependence study. The emission spectrum of pristine $(6,5)$ SWCNT (black) is included for reference. 


\section{Evaluation of photo-induced electron transfer mechanism.}

Apparent quantum efficiency (AQE), a measure of the efficiency of the conversion of absorbed incident photons to covalent bonding, was calculated using our defect PL intensities at $\mathrm{E}_{11}{ }^{-}$(565 nm exc.; see Figure S6). The resulting plot of AQE versus irradiation energy was overlaid with the optical absorption spectrum of the SWCNT as shown below.

$$
A Q E=\frac{E_{11}^{-}(\lambda)-E_{11}^{-}(\text {dark })}{\text { no. of incident photons }}
$$

The number of incident photons was calculated based on the incident irradiation power which was measured by a calibrated power meter. The incident power densities at 312 and $345 \mathrm{~nm}$ were extrapolated based on the output spectrum of the Xe arc lamp because these energies lie outside of the power meter's calibration range. All AQE values were normalized to the highest value for simplicity.

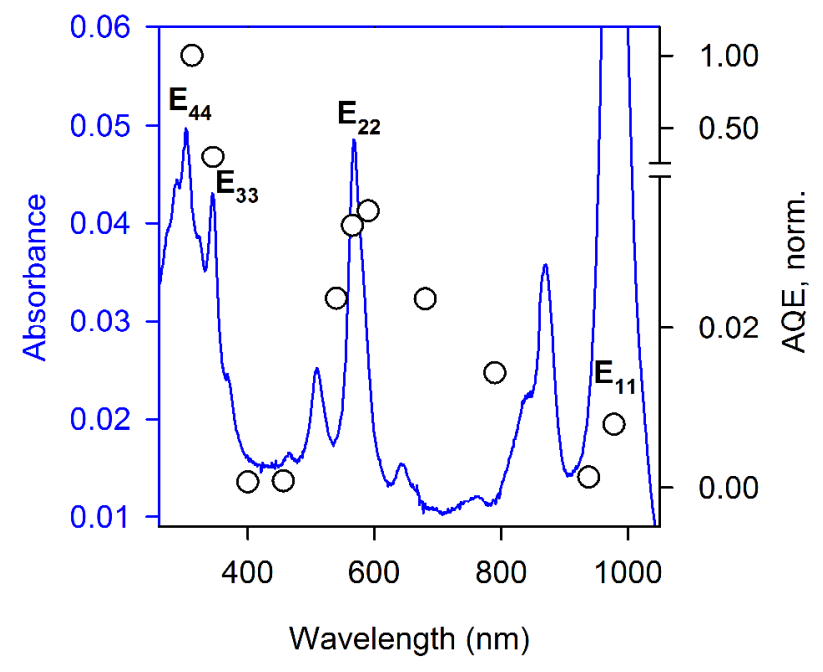

Figure S6. Apparent quantum efficiency (open circles) as a function of irradiation wavelength is compared to the optical absorption spectrum of $(6,5)-\mathrm{SWCNT}$ (blue line). 